\title{
Article \\ Shoulder Kinematics and Symmetry at Different Load Intensities during Bench Press Exercise
}

\author{
Juan Manuel Franco-García ${ }^{1,2, *(D)}$, Miguel Rodal ${ }^{1} \mathbb{1}$, Rafael Gutiérrez-Horrillo ${ }^{1}$, Jorge Carlos-Vivas ${ }^{2} \oplus$, \\ Jorge Pérez-Gómez ${ }^{2, *}$, Hadi Nobari ${ }^{2,3}$, Luca Paolo Ardigò ${ }^{4, *,+}$ and Konstantinos Gianikellis $1,+$ \\ 1 BioẼrgon Research Group, Faculty of Sports Sciences, University of Extremadura, 10003 Cáceres, Spain; \\ mrodal@unex.es (M.R.); rgutierris@alumnos.unex.es (R.G.-H.); kgiannik@unex.es (K.G.) \\ 2 Health, Economy, Motricity and Education Research Group (HEME), Faculty of Sports Sciences, \\ University of Extremadura, 10003 Cáceres, Spain; jorgecv@unex.es (J.C.-V.); \\ hnobarin@alumnos.unex.es (H.N.) \\ 3 Department of Physical Education and Sports, University of Granada, 18010 Granada, Spain \\ 4 Department of Neurosciences, Biomedicine and Movement Sciences, School of Exercise and Sport Science, \\ University of Verona, 37131 Verona, Italy \\ * Correspondence: jufrancog@alumnos.unex.es (J.M.F.-G.); jorgepg100@unex.es (J.P.-G.); \\ luca.ardigo@univr.it (L.P.A.) \\ + These authors share last authorship.
}

\section{check for} updates

Citation: Franco-García, J.M.; Rodal, M.; Gutiérrez-Horrillo, R.; Carlos-Vivas, J.; Pérez-Gómez, J.; Nobari, H.; Ardigò, L.P.; Gianikellis, K. Shoulder Kinematics and Symmetry at Different Load Intensities during Bench Press Exercise. Symmetry 2021, 13, 1859. https://doi.org/10.3390/sym13101859

Academic Editor: Alejandro

F Villaverde

Received: 1 July 2021

Accepted: 2 September 2021

Published: 3 October 2021

Publisher's Note: MDPI stays neutral with regard to jurisdictional claims in published maps and institutional affiliations.

Copyright: (c) 2021 by the authors. Licensee MDPI, Basel, Switzerland. This article is an open access article distributed under the terms and conditions of the Creative Commons Attribution (CC BY) license (https:// creativecommons.org/licenses/by/ $4.0 /)$.
Abstract: This study aimed to analyze between-shoulder kinematics symmetry at different load intensities considering full range of movement (ROM), mean and maximum velocities ( $V_{\text {MEAN }}$, $\mathrm{V}_{\mathrm{MAX}}$ ), and accelerations ( $\mathrm{A}_{\mathrm{MEAN}}, \mathrm{A}_{\mathrm{MAX}}$ ) of shoulders during phases 2 (characterized by positive acceleration and negative velocity, eccentric) and 3 (characterized by positive acceleration and velocity, concentric) of bench press exercise (BP); as well as to compare unilateral kinematics variables between the different load intensity intervals. Twenty-seven participants were evaluated during phases 2 and 3 of BP at different load intervals: interval 1 (55-75\% 1-repetition maximum: 1RM), interval 2 (75-85\% 1RM) and interval 3 (85-100\% 1RM). Kinematics variables were determined using the Xsens MVN Link System. Results showed that full ROM was higher in left than right shoulder at all intensities $(p=0.008-0.035)$. $\mathrm{V}_{\text {MEAN }}, \mathrm{V}_{\mathrm{MAX}}, \mathrm{A}_{\mathrm{MEAN}}$, and $\mathrm{A}_{\mathrm{MAX}}$ were different in both shoulders for interval 3 during phase 2 and were lower as load intensity increased in both shoulders ( $p=0.001-0.029$ ). During phase 3, only $\mathrm{V}_{\mathrm{MAX}}$ on interval 2 was different between shoulders. Moreover, $\mathrm{V}_{\text {MEAN }}, \mathrm{V}_{\mathrm{MAX}}, \mathrm{A}_{\mathrm{MEAN}}$, and $\mathrm{A}_{\mathrm{MAX}}$ were greater during interval 1 compared with the others in both shoulders $(p=0.001-0.029)$. Therefore, there exists a kinematics asymmetry between both shoulders during phases 2 and 3 of bench press, although the acceleration was similar during both phases at all load intensities. Moreover, kinematic parameters differ between loads of $55-75 \%$ RM compared to $75-100 \%$ RM loads.

Keywords: acceleration; biomechanics; full ROM; shoulder; velocity

\section{Introduction}

Bench press (BP) is one of the most popular overload training exercises in different disciplines (e.g., weightlifting, powerlifting, etc.). Specifically, in powerlifters, it is the main exercise for measuring maximal upper body strength during a competition, just as happens with squat and deadlift for lower body [1]. Moreover, the BP exercise is usually used to induce hypertrophy and enhance muscle power [2,3]. Measuring how the peak load descends to the chest and ascends to full elbow extension is important to understand the kinematic parameters and performance factors of the BP exercise [4]. Although several publications have addressed BP and bar kinematics, there is a lack of research on shoulder kinematics during the full execution of this exercise. Krzysztofik et al. (2020) [5] compared the electromyographic activity between the standard and cambered bar during BP, observing that a superior ROM due to the use of cambered bar increased anterior deltoid muscle 
activation compared to the standard bar, which showed higher pectoralis major and triceps brachii long head activation during BP.

The analysis of joint kinematics allows us to quantify the amplitude of the range of motion (ROM) [6] to prevent injuries and improve athletes' performance [7]. Specifically, shoulder injuries are the most common in weightlifters [8] since the shoulder joint is exposed to repetitive stresses and high weights during $\mathrm{BP}$, as well as connective tissue changes and joint instability [9]. Thus, it could be interesting knowing the articular kinematics of shoulder to direct injury prevention strategies.

The main performance parameters of BP studied are full ROM, $\mathrm{V}_{\text {MEAN }}, \mathrm{V}_{\mathrm{MAX}}$ [10-13], $\mathrm{A}_{\mathrm{MEAN}}$, and $\mathrm{A}_{\mathrm{MAX}}$ [12]; in addition to muscle activity or sticking region. However, these variables have been frequently measured on the bar, being scantly analyzed at joint level (e.g., shoulder, elbow, and so on) [14-16].

Performance during BP exercise has been analyzed using different systems, and devices such as linear transducers have been used to examine the behavior of the bar during execution [17,18], as well as 3D motion analysis systems [19-21] or electronic goniometer [22] to observe the behavior of both the bar and the joints involved in this exercise. Castro et al. (2020) [19] studied the aforementioned variables using 3D systems, but they only analyzed the kinematics of BP using markers on the bar without considering the analysis of the shoulder joint. In another way, Ojasto and Hakkinen (2009) [22] measured joint displacement and velocity of the elbow joint using 3D systems, reporting significant differences during the eccentric and concentric phase as the load increased $(p<0.01)$; however, it is unknown what happens with the shoulder joint. In contrast, Larsen et al. (2021) [23] analyzed the shoulder joint velocity during the concentric phase of BP using 3D cameras, finding significant differences in the shoulder (flexion or adduction) as a function of the type of grip used in the BP exercise (wide, normal, and narrow) $(p<0.05)$, but it was not considered the eccentric phase of exercise. None of these previous studies analyzed the acceleration variable, even though it might be a key result for changing the barbell direction since it is well-known that for modifying velocity, an acceleration is needed; additionally, acceleration will only occur if there is an application of a moment of force. Similarly, it has not been studied whether there is symmetry between the left and right shoulder in terms of these variables. In contrast, other research has addressed symmetry in BP exercise by electromyographic analysis [24,25]; Krzysztofik et al. (2021) [24] indicated that there is greater muscle activity in the anterior deltoid and triceps brachii on the dominant side $(p<0.001)$, but joint kinematics as a factor influencing muscle electrical activity was not studied. As an alternative to analyze the symmetry in BP, the Xsens system used in this study allows real-time measurement of the variables: ROM, $V_{\text {MEAN }}, V_{M A X}, A_{M E A N}$, and $\mathrm{A}_{\mathrm{MAX}}$, during the complete execution of the BP exercise, as well as being time saving in documenting the subject.

Recent research, by analyzing the acceleration and power curves of the barbell, determined that the BP consists of four phases, and of those phases, 2 and 3 of this exercise are the most relevant (Figure 1): During phase 1, there was a negative vertical acceleration and velocity of the barbell so that the mechanical power was positive (i.e., concentric). However, it was observed that the movement of the barbell was due to the attraction of gravity and not to the concentric action of elbow flexors and shoulder extensors. Phase 2 was characterized by positive acceleration combined with negative velocity, which indicated the performance of eccentric mechanical power and eccentric mechanical work of the elbow extensors and shoulder flexors. During phase 3, there was positive acceleration combined with positive velocity, which indicated the performance of the elbow extensors and shoulder flexors, generating concentric mechanical power. Finally, phase 4 was characterized by negative acceleration with positive velocity, which would involve the eccentric power of the elbow flexors and shoulder extensors, but it is apparent that the movement of the barbell is due, mainly, to the attraction of gravity and not to the muscle performance, even though muscle activity plays a part in the development of the action. 

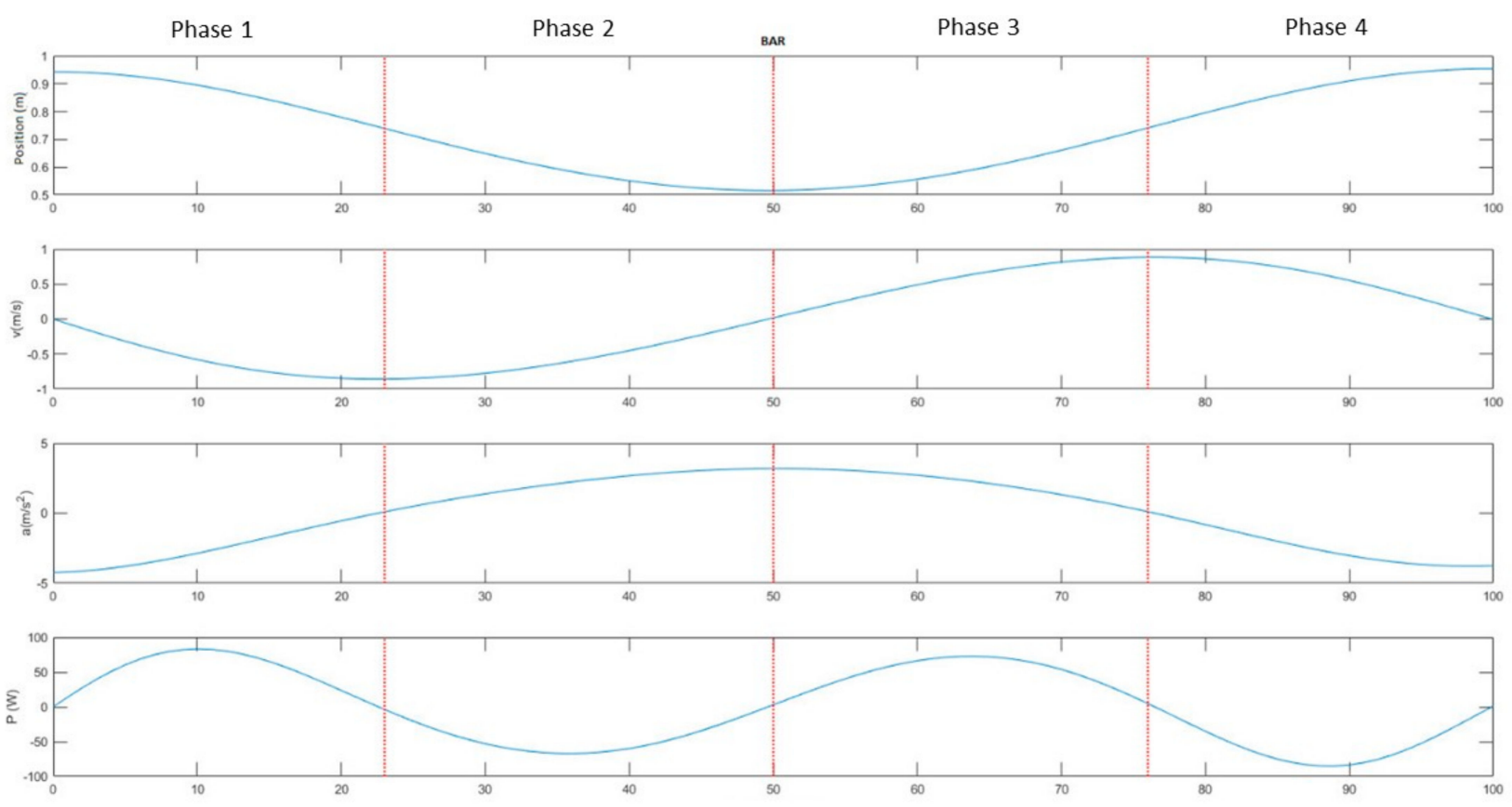

Figure 1. Bench press phases based on acceleration and power curves of the barbell analyses.

Based on the above, it would be interesting to examine the shoulder articulation during BP exercise in phases 2 and 3, the symmetry between both shoulders based on different load intensities applied, and how it might influence performance at different intensities. Therefore, this study aimed (1) to analyze the symmetry between the right and left shoulders at different load intensities considering full ROM, mean and maximum velocity, and acceleration of shoulder during both phases 2 and 3 of BP; and (2) to compare these kinematic parameters between the different load intensity intervals on each shoulder, separately.

We hypothesized that (1) there will exist symmetry between shoulders independent of load intensities considering full ROM, mean and maximum velocity, and acceleration of shoulder during phase 2 and phase 3 of BP; and (2) $V_{M A X}, V_{\text {MEAN }}, A_{M A X}$, and AMEAN will be lower as load intensity increases in both shoulders during phases 2 and 3 of BP, while full ROM will maintain constant for all load intensities.

\section{Materials and Methods}

\subsection{Study Design}

A cross-sectional study was conducted to analyze and to compare the differences and symmetry in these parameters among different intensity intervals in BP exercise: Interval 1 (55-75\% RM), Interval 2 (75-85\% RM), and interval 3 (85-100\% RM). The following parameters were computed for phases 2 and 3 under different load conditions: (1) full ROM; (2) V $\mathrm{V}_{\text {MAX }}$; (3) $\mathrm{V}_{\text {MEAN }}$; (4) A MAX; (5) A AEAN. To collect these data, a directcomputation 1-repetition maximum (1RM) protocol of the BP was followed. The shoulder kinematics was recorded for each lift during 1RM protocol at every load intensity applied.

\subsection{Ethical Approval}

The study procedures and informed consent were approved by the Institutional Bioethics and Biosafety Committee of the University of Extremadura in accordance with the Declaration of Helsinki (registration number: 126/2018).

\subsection{Participants}

Twenty-seven healthy recreational weightlifters were recruited for this study: 17 men (age: 21.4 (1.5) years; height: 175.1 (6.7) cm; body mass: 75.8 (7.7) kg; BP 1RM: 91.7 (13.7) kg) 
and 10 women (age: 21.7 (2.3) years; height: $163.3(10.8) \mathrm{cm}$; body mass: 57.2 (6.8) kg; BP 1RM: $38.9(10.5) \mathrm{kg}$ ). To be included into the study, they needed to meet the following eligibility criteria: (1) age between 18 and 40 years, (2) at least 1 year of experience in recreational weightlifting, (3) regular practice of BP (two or more days per week), (4) no upper trunk injury in the last year, and (5) no medical contraindication to perform maximal effort. All participants were informed about the research procedures and signed the informed consent before starting the study.

\subsection{Procedures}

A standardized 1RM protocol was conducted, following the guidelines previously described by Gregory and Travis (2015) [4]. Thus, a cardiovascular warm-up was performed (5-10 $\mathrm{min})$, then the subjects performed a set of 5 to 10 easy repetitions in the BP exercise, recovered one minute, and added 4 to $9 \mathrm{~kg}$ to perform 3 to 5 repetitions. After resting $2 \mathrm{~min}$, they again added 4 to $9 \mathrm{~kg}$ to perform 2 to 3 repetitions. After $4 \mathrm{~min}$ of recovery they added 4 to $9 \mathrm{~kg}$ to perform 1 repetition. After a $5 \mathrm{~min}$ rest, they added weight under the participant's perception. They were encouraged to raise the barbell at maximum velocity. A strength and conditioning coach supervised all participants during warm-up and every BP set to check and ensure that the movement pattern was performed properly. Then, the sets in which subjects worked at the established ranges of intensities were noted and compared.

The Xsens MVN Link system and the MVN Analyze 2018 software (Enchende, The Netherlands) were used to obtain data and record the kinematics of the whole dynamic system with the load [26]. The Xsens device sampling rate was $240 \mathrm{~Hz}$. These data were carefully stored for subsequent digital processing. Position $(\vec{x})$, angular velocity $(\vec{\omega})$, and angular acceleration $(\vec{a})$ of shoulder joint were calculated through the data obtained from the sensor placed on the back of the scapula, which registers the joint position. The calibration procedure followed was the T-pose + walk mode. Then, participants started lying on the bench with feet flat on the floor, arms extended at shoulder width, while holding the bar and shoulders at 90 degrees. Moreover, the middle point of the barbell was determined, and from there, participants placed their hands shoulder-width apart, maintaining an equal separation from the barbell middle point. Position data was derived to obtain the velocity and acceleration data, respectively. To analyze the repetitions, the values of the central repetitions were taken for each series, representing the median of the series. These data underwent a smoothing process using quintic splines [27]. This real-time inertial measurement system has shown to be valid and reliable for analyzing flexion and extension of the variable position, velocity, and acceleration in shoulder joint intraclass correlation (ICC) 0.71 to 0.99 [28].

\subsection{Statistical Analysis}

Statistical procedures and computations were conducted using SPSS (version 25.0; IBM SPSS Inc, Armonk, NY, USA). Data are presented as mean and standard deviation (SD). Normality and homogeneity of data were checked applying Shapiro-Wilk and Levene's tests, respectively. A two-way (load intensity $\times$ laterality) repeated measures analysis of variance (ANOVA) was carried out to analyze all dependent variables during both phases 2 and 3 of BP. Partial eta squared $\left(\eta p^{2}\right)$ was used as effect size for multiple-comparisons ANOVA analysis. Then, Bonferroni post hoc test was applied for pairwise comparisons considering both load intensity intervals and laterality. Hedges' $g$ effect size was also calculated for pairwise comparisons. Hedges' $g$ thresholds were determined as follows: $\leq 0.2$, trivial; $>0.2$, small; $>0.6$, moderate; $>1.2$, large; $>2.0$, very large; and $>4.0$, nearly perfect [29]. Significant differences were considered for $p \leq 0.05$. Despite the small sample size, a total of 27 participants were enough to reach a power of $88 \%$, considering a 0.25 effect size $f$ and alpha level equal to 0.05 and a 0.6 correlation among repeated measures. 


\section{Results}

Multiple comparisons ANOVA revealed a significant main effect of laterality $(p=0.006$; $\eta p^{2}=0.252$ ). Figure 2 shows the comparison between left and right shoulders for ROM at the different load intensities during BP. Specifically, pairwise comparisons showed a significantly greater ROM of left shoulder compared to right shoulder at all load intensity intervals (Interval 1: $p=0.008, g=0.52$; Interval 2: $p=0.035, g=0.46$; Interval 3: $p=0.015$, $g=0.46)$. No effect of load intensity nor load intensity $\mathrm{x}$ laterality was observed $(p>0.05)$.

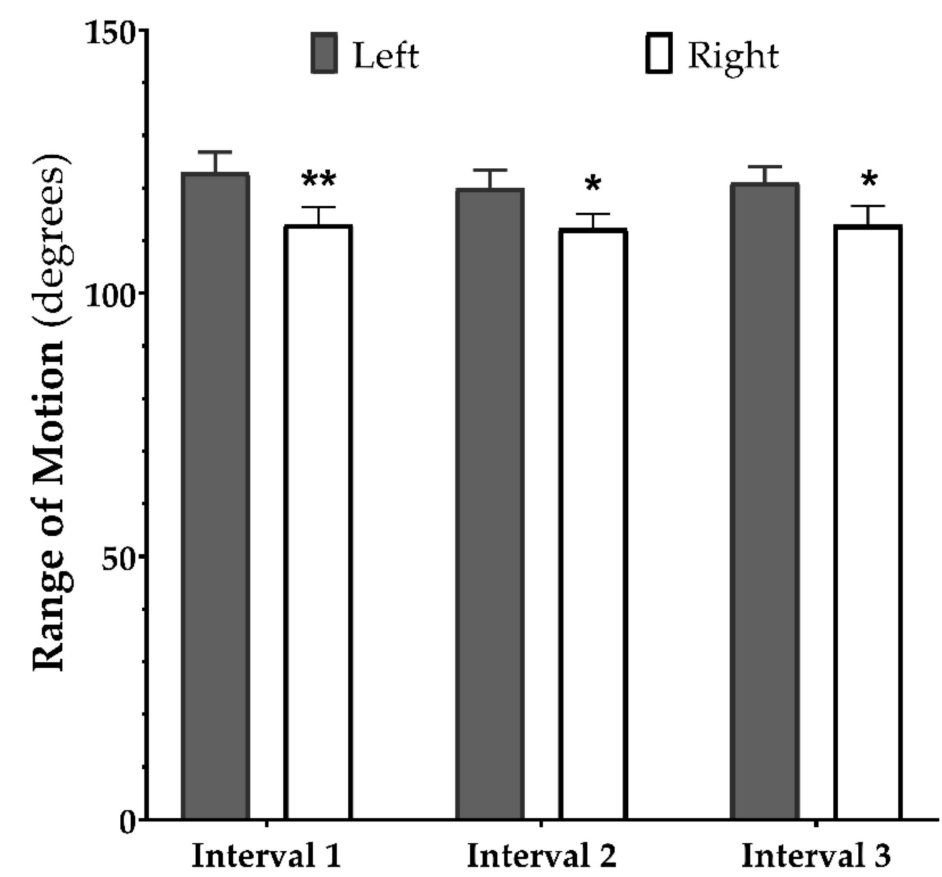

Figure 2. Between-shoulder comparison for ROM at different load intensities during bench press. * Significant differences for $p \leq 0.05$. ${ }^{*}$ Significant differences for $p \leq 0.01$.

Multiple comparisons ANOVA revealed a significant main effect of laterality in $\mathrm{V}_{\mathrm{MAX}}$ $\left(p=0.018 ; \eta p^{2}=0.197\right)$ and $\operatorname{VMEAN}_{\text {ME }}\left(p=0.015 ; \eta p^{2}=0.206\right)$. There was no effect of laterality in $A_{\text {MAX }}$ and $A_{\text {MEAN }}(p>0.05)$. There was also an effect of load intensity on $V_{\text {MAX }}(p<0.001$; $\left.\eta \mathrm{p}^{2}=0.549\right), \mathrm{V}_{\text {MEAN }}\left(p<0.001 ; \eta \mathrm{p}^{2}=0.486\right), \mathrm{A}_{\text {MAX }}\left(p<0.001 ; \eta \mathrm{p}^{2}=0.538\right)$, and $\mathrm{A}_{\text {MEAN }}$ $\left(p=0.008 ; \eta p^{2}=0.321\right)$. However, there were no effects on laterality $\mathrm{x}$ intensity in any parameter $(p>0.05)$.

Table 1 shows the comparison between right and left shoulders in kinematic parameters for the different intensity intervals during phase 2 . Results revealed significant differences between both shoulders for the interval 3 in $V_{M A X}$ and $V_{\text {MEAN }}$ during phase 2 of BP. However, no differences were found for intervals 1 and $2(p>0.05)$. No differences were also observed in $\mathrm{A}_{\text {MAX }}$ and $\mathrm{A}_{\text {MEAN }}(p>0.05)$ for any intensity interval comparing both shoulders in this phase.

Tables 2 and 3 show the comparison among intensity intervals on kinematic parameters for left and right shoulders, respectively, during phase 2. Coincidently, $\mathrm{V}_{\text {MAX }}, \mathrm{V}_{\text {MEAN }}$, $A_{M A X}$, and $A_{M E A N}$ were significantly greater during interval 1 compared to interval 2 in both left and right shoulders during phase 2 . Similarly, all these parameters were higher in interval 1 compared to interval 3 in both shoulders, except $A_{\text {MEAN }}$ in left shoulder $(p>0.05)$. However, no differences were observed in any variables when intervals 2 and 3 were compared $(p>0.05)$. 
Table 1. Between-shoulder comparison for kinematic parameters at different load intensities during the phase 2 of bench press.

\begin{tabular}{|c|c|c|c|c|c|}
\hline Parameter & Interval & $\begin{array}{l}\text { Left Shoulder } \\
\text { Mean (SD) }\end{array}$ & $\begin{array}{l}\text { Right Shoulder } \\
\text { Mean (SD) }\end{array}$ & $p$ & $\begin{array}{c}\text { Hedges' } \\
g(95 \% \text { CI })\end{array}$ \\
\hline \multirow{3}{*}{$\begin{array}{c}\mathbf{V}_{\text {MAX }} \\
\left(\mathrm{deg} \cdot \mathrm{s}^{-1}\right)\end{array}$} & 1 & $238.07(13.36)$ & $221.13(11.82)$ & 0.080 & $1.32(0.73 ; 1.91)$ \\
\hline & 2 & $197.10(9.90)$ & $173.24(10.16)$ & 0.060 & $2.34(1.65 ; 3.04)$ \\
\hline & 3 & $166.72(10.65)$ & 150.58 (10.66) & 0.046 & $1.49(0.89 ; 2.10)$ \\
\hline \multirow{3}{*}{$\begin{array}{l}\text { V }_{\text {MEAN }} \\
\left(\mathrm{deg} \cdot \mathrm{s}^{-1}\right)\end{array}$} & 1 & $144.42(8.24)$ & $131.78(7.56)$ & 0.057 & $1.58(0.96 ; 2.19)$ \\
\hline & 2 & $121.72(5.56)$ & $103.86(9.70)$ & 0.089 & $2.23(1.55 ; 2.91)$ \\
\hline & 3 & $107.77(6.57)$ & $96.85(6.22)$ & 0.026 & $1.68(1.06 ; 2.30)$ \\
\hline \multirow{3}{*}{$\underset{\left(\mathrm{deg} \cdot \mathrm{s}^{-2}\right)}{\mathbf{A}_{\mathbf{M A X}}}$} & 1 & $\begin{array}{l}1208.63 \\
(101.29)\end{array}$ & 1145.86 (91.73) & 0.363 & $0.64(0.09 ; 1.19)$ \\
\hline & 2 & $874.39(62.2)$ & $800.03(67.41)$ & 0.243 & $1.13(0.56 ; 1.70)$ \\
\hline & 3 & 735.03 (72.76) & 664.90 (70.99) & 0.076 & $0.96(0.40 ; 1.52)$ \\
\hline \multirow{3}{*}{ 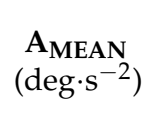 } & 1 & 498.22 (90.78) & 468.20 (83.19) & 0.514 & $0.34(-0.20 ; 0.88)$ \\
\hline & 2 & $341.34(63.41)$ & 309.97 (67.85) & 0.450 & $0.47(-0.07 ; 1.01)$ \\
\hline & 3 & $335.93(64.17)$ & 301. $15(57.64)$ & 0.175 & $0.56(0.02 ; 1.11)$ \\
\hline
\end{tabular}

$\mathrm{A}_{\mathrm{MAX}}$, maximum acceleration in deg. $\mathrm{s}^{-2} ; \mathrm{A}_{\mathrm{MEAN}}$, mean acceleration in deg. $\mathrm{s}^{-2} ; \mathrm{V}_{\mathrm{MAX}}$, maximum velocity in $\mathrm{deg} \cdot \mathrm{s}^{-1} ; \mathrm{V}_{\mathrm{MEAN}}$, mean velocity in deg. $\mathrm{s}^{-1}$. Significant differences for $p$-value $\leq 0.05$ are highlighted in bold.

Table 2. Comparison between load intensity intervals for kinematic parameters in the left shoulder during phase 2 of bench press.

\begin{tabular}{|c|c|c|c|c|}
\hline Parameter & $\begin{array}{c}\text { Interval } \\
\text { Mean (SD) }\end{array}$ & Comparative & $p$ & $\begin{array}{c}\text { Hedges' } \\
g(95 \% \text { CI })\end{array}$ \\
\hline \multirow{3}{*}{$\mathbf{V}_{\text {MAX }}\left(\mathrm{deg} \cdot \mathrm{s}^{-1}\right)$} & Int 1: -238.07 (13.36) & 1 vs. 2 & 0.026 & $3.43(2.59 ; 4.27)$ \\
\hline & Int 2: $-197.10(9.90)$ & 1 vs. 3 & 0.001 & $5.82(4.60 ; 7.04)$ \\
\hline & Int 3: $-166.72(10.65)$ & 2 vs. 3 & 0.055 & $2.91(2.15 ; 3.68)$ \\
\hline \multirow{3}{*}{$\begin{array}{l}\text { V MEAN }_{\text {MEA }} \\
\left(\text { deg } \cdot s^{-1}\right)\end{array}$} & Int $1:-144.42(8.24)$ & 1 vs. 2 & 0.013 & $3.18(2.38 ; 3.99)$ \\
\hline & Int $2:-121.72(5.56)$ & 1 vs. 3 & 0.002 & $4.85(3.79 ; 5.91)$ \\
\hline & Int $3:-107.77$ (6.57) & 2 vs. 3 & 0.215 & $2.26(1.58 ; 2.94)$ \\
\hline \multirow{3}{*}{$\mathbf{A}_{\text {MAX }}\left(\mathrm{deg} \cdot \mathrm{s}^{-2}\right)$} & Int 1: 1208.63 (101.29) & 1 vs. 2 & 0.013 & $3.92(3.01 ; 4.83)$ \\
\hline & Int 2: $874.39(62.2)$ & 1 vs. 3 & 0.001 & $5.29(4.16 ; 6.42)$ \\
\hline & Int 3: 735.03 (72.76) & 2 vs. 3 & 0.151 & $2.03(1.37 ; 2.69)$ \\
\hline \multirow{3}{*}{$\begin{array}{c}\text { A MEAN }_{\left(\mathrm{deg} \cdot \mathrm{s}^{-2}\right)} \\
\end{array}$} & Int 1: 498.22 (90.78) & 1 vs. 2 & 0.027 & $1.97(1.32 ; 2.63)$ \\
\hline & Int 2: 341.34 (63.41) & 1 vs. 3 & 0.054 & $2.03(1.38 ; 2.69)$ \\
\hline & Int 3: 335.93 (64.17) & 2 vs. 3 & 1.000 & $0.08(-0.45 ; 0.62)$ \\
\hline
\end{tabular}

$\mathrm{A}_{\mathrm{MAX}}$, maximum acceleration in $\mathrm{deg} \cdot \mathrm{s}^{-2} ; \mathrm{A}_{\mathrm{MEAN}}$, mean acceleration in $\mathrm{deg} \cdot \mathrm{s}^{-2} ; \mathrm{V}_{\mathrm{MAX}}$, maximum velocity in deg. $\mathrm{s}^{-1} ; \mathrm{V}_{\text {MEAN }}$, mean velocity in deg. $\mathrm{s}^{-1}$. Significant differences for $p$-value $\leq 0.05$ are highlighted in bold.

Multiple comparisons ANOVA also revealed a significant main effect of laterality in $\mathrm{V}_{\text {MAX }}\left(p=0.020 ; \eta p^{2}=0.192\right)$ and $\mathrm{V}_{\text {MEAN }}\left(p=0.036 ; \eta p^{2}=0.158\right)$. However, there was no effect of laterality in $\mathrm{A}_{\mathrm{MAX}}$ and $\mathrm{A}_{\text {MEAN }}(p>0.05)$. Moreover, there was an effect of load intensity on $\mathrm{V}_{\mathrm{MAX}}\left(p<0.001 ; \eta \mathrm{p}^{2}=0.744\right), \mathrm{V}_{\text {MEAN }}\left(p<0.001 ; \eta \mathrm{p}^{2}=0.722\right), \mathrm{A}_{\mathrm{MAX}}(p<0.001$; $\left.\eta \mathrm{p}^{2}=0.542\right)$, and $\mathrm{A}_{\text {MEAN }}\left(p<0.001 ; \eta \mathrm{p}^{2}=0.637\right)$. However, there were no effects on laterality $\mathrm{x}$ intensity in any parameter $(p>0.05)$.

Table 4 presents the comparative analysis between left and right shoulders in kinematic parameters for the different intensity intervals in phase 3 . Results revealed no significant differences between both shoulders in any variables, except $\mathrm{V}_{\mathrm{MAX}}$ of interval 2. 
Table 3. Comparison between load intensity intervals for kinematic parameters in the right shoulder during phase 2 of bench press.

\begin{tabular}{|c|c|c|c|c|}
\hline Parameter & $\begin{array}{c}\text { Interval } \\
\text { Mean (SD) }\end{array}$ & Comparative & $p$ & $\begin{array}{c}\text { Hedges' } \\
g(95 \% \text { CI })\end{array}$ \\
\hline \multirow{3}{*}{$\mathbf{V}_{\text {MAX }}\left(\mathrm{deg} \cdot \mathrm{s}^{-1}\right)$} & Int 1: $-221.13(11.82)$ & 1 vs. 2 & 0.001 & $4.28(3.31 ; 5.25)$ \\
\hline & Int 2: $-173.24(10.16)$ & 1 vs. 3 & $<0.001$ & $6.18(4.90 ; 7.46)$ \\
\hline & Int 3: $-150.58(10.66)$ & 2 vs. 3 & 0.166 & $2.14(1.48 ; 2.81)$ \\
\hline \multirow{3}{*}{$\begin{array}{l}\text { V MEAN }_{\text {MEA }} \\
\left(\mathrm{deg} \cdot \mathrm{s}^{-1}\right)\end{array}$} & Int $1:-131.78(7.56)$ & 1 vs. 2 & 0.029 & $3.16(2.36 ; 3.96)$ \\
\hline & Int 2: $-103.86(9.70)$ & 1 vs. 3 & $<0.001$ & $4.97(3.89 ; 6.05)$ \\
\hline & Int 3: $-96.85(6.22)$ & 2 vs. 3 & 1.000 & $0.85(0.29 ; 1.40)$ \\
\hline \multirow{3}{*}{$\mathbf{A}_{\text {MAX }}\left(\mathrm{deg} \cdot \mathrm{s}^{-2}\right)$} & Int 1: $1145.86(91.73)$ & 1 vs. 2 & $<0.001$ & $4.23(3.27 ; 5.19)$ \\
\hline & Int 2: 800.03 (67.41) & 1 vs. 3 & $<0.001$ & $5.78(4.57 ; 6.99)$ \\
\hline & Int 3: 664.90 (70.99) & 2 vs. 3 & 0.144 & $1.92(1.28 ; 2.57)$ \\
\hline \multirow{3}{*}{$\begin{array}{c}\text { A MEAN }_{\left(\mathrm{deg} \cdot \mathrm{s}^{-2}\right)} \\
\left({ }^{-2}\right.\end{array}$} & Int 1: 468.20 (83.19) & 1 vs. 2 & 0.010 & $2.05(1.39 ; 2.71)$ \\
\hline & Int 2: 309.97 (67.85) & 1 vs. 3 & 0.003 & $2.30(1.61 ; 2.99)$ \\
\hline & Int 3: 301.15 (57.64) & 2 vs. 3 & 1.000 & $0.14(-0.40 ; 0.67)$ \\
\hline
\end{tabular}

$\mathrm{A}_{\mathrm{MAX}}$, maximum acceleration in deg $\cdot \mathrm{s}^{-2} ; \mathrm{A}_{\mathrm{MEAN}}$, mean acceleration in deg $\cdot \mathrm{s}^{-2} ; \mathrm{V}_{\mathrm{MAX}}$, maximum velocity in $\mathrm{deg} \cdot \mathrm{s}^{-1} ; \mathrm{V}_{\mathrm{MEAN}}$, mean velocity in deg. $\mathrm{s}^{-1}$. Significant differences for $p$-value $\leq 0.05$ are highlighted in bold.

Table 4. Between-shoulder comparison for kinematic parameters at different load intensities during phase 3 of bench press.

\begin{tabular}{cccccc}
\hline Parameter & Interval & $\begin{array}{c}\text { Left Shoulder } \\
\text { Mean (SD) }\end{array}$ & $\begin{array}{c}\text { Right Shoulder } \\
\text { Mean (SD) }\end{array}$ & $p$ & $\begin{array}{c}\text { Hedges' } \\
\boldsymbol{( 9 5 \%} \text { CI) }\end{array}$ \\
\hline $\mathbf{V}_{\text {MAX }}$ & 1 & $250.16(11.44)$ & $230.37(11.25)$ & 0.058 & $1.72(1.09 ; 2.34)$ \\
$\left(\mathrm{deg} \cdot \mathrm{s}^{-1}\right)$ & 2 & $194.93(8.72)$ & $172.53(10.66)$ & $\mathbf{0 . 0 5 0}$ & $2.27(1.58 ; 2.95)$ \\
& 3 & $151.62(12.19)$ & $139.56(11.72)$ & 0.142 & $0.99(0.43 ; 1.65)$ \\
\hline $\mathbf{V}_{\text {MEAN }}$ & 1 & $152.17(7.06)$ & $139.53(6.57)$ & 0.068 & $1.83(1.19 ; 2.46)$ \\
$\left(\mathrm{deg} \cdot \mathrm{s}^{-1}\right)$ & 2 & $120.60(5.08)$ & $106.75(9.11)$ & 0.126 & $1.85(1.21 ; 2.49)$ \\
& 3 & $95.92(7.11)$ & $88.45(7.39)$ & 0.144 & $1.02(0.45 ; 1.58)$ \\
\hline $\mathbf{A}_{\text {MAX }}$ & 1 & $1212.93(100.70)$ & $1146.49(92.51)$ & 0.339 & $0.68(0.13 ; 1.23)$ \\
$\left(\mathrm{deg} \cdot \mathrm{s}^{-2}\right)$ & 2 & $868.30(63.12)$ & $799.73(67.92)$ & 0.279 & $1.03(0.46 ; 1.60)$ \\
& 3 & $735.58(72.98)$ & $660.96(71.63)$ & 0.062 & $1.02(0.45 ; 1.58)$ \\
\hline $\mathbf{A}_{\text {MEAN }}$ & 1 & $701.06(52.82)$ & $641.74(51.71)$ & 0.232 & $1.12(0.54 ; 1.69)$ \\
$\left(\mathrm{deg} \cdot \mathrm{s}^{-2}\right)$ & 2 & $488.40(36.71)$ & $436.71(49.54)$ & 0.200 & $1.17(0.59 ; 1.75)$ \\
& 3 & $368.00(52.12)$ & $320.64(55.26)$ & 0.177 & $0.87(0.31 ; 1.43)$ \\
\hline
\end{tabular}

$\mathrm{A}_{\mathrm{MAX}}$, maximum acceleration in deg. $\mathrm{s}^{-2} ; \mathrm{A}_{\mathrm{MEAN}}$, mean acceleration in $\mathrm{deg} \cdot \mathrm{s}^{-2} ; \mathrm{V}_{\mathrm{MAX}}$, maximum velocity in $\mathrm{deg} \cdot \mathrm{s}^{-1} ; \mathrm{V}_{\mathrm{MEAN}}$, mean velocity in deg. $\mathrm{s}^{-1}$. Significant differences for $p$-value $\leq 0.05$ are highlighted in bold.

Tables 5 and 6 illustrate the between-interval comparison for kinematic parameters for left and right shoulders, respectively, during phase 3 . As expected, $V_{M A X}, V_{\text {MEAN }}, A_{M A X}$, and $A_{\text {MEAN }}$ were significantly greater during interval 1 compared to intervals 2 and 3 in both shoulders during phase 3 . However, only $\mathrm{V}_{\mathrm{MAX}}$ was higher in interval 2 compared to interval 3 for both shoulders. 
Table 5. Comparison between load intensity intervals for kinematic parameters in the left shoulder during phase 3 of bench press.

\begin{tabular}{|c|c|c|c|c|}
\hline Parameter & Interval & Comparative & $p$ & $\begin{array}{c}\text { Hedges' } \\
g(95 \% \text { CI })\end{array}$ \\
\hline \multirow{3}{*}{$\mathbf{V}_{\text {MAX }}\left(\mathrm{deg} \cdot \mathrm{s}^{-1}\right)$} & Int 1: 250.16 (11.44) & 1 vs. 2 & $<0.001$ & $8.22(6.58 ; 9.85)$ \\
\hline & Int 2: 194.93 (8.72) & 1 vs. 3 & $<0.001$ & $9.41(7.56 ; 11.27)$ \\
\hline & Int 3: 151.62 (12.19) & 2 vs. 3 & 0.009 & $0.99(0.43 ; 1.56)$ \\
\hline \multirow{3}{*}{$\begin{array}{l}\text { V MEAN }_{\text {MEA }} \\
\left(\text { deg } \cdot \mathrm{s}^{-1}\right)\end{array}$} & Int 1: 152.17 (7.06) & 1 vs. 2 & 0.013 & $7.82(6.26 ; 9.39)$ \\
\hline & Int 2: 120.60 (5.08) & 1 vs. 3 & 0.002 & $8.69(6.97 ; 10.41)$ \\
\hline & Int 3: 95.92 (7.11) & 2 vs. 3 & 0.215 & $1.02(0.45 ; 1.58)$ \\
\hline \multirow{3}{*}{$\mathbf{A}_{\text {MAX }}\left(\mathrm{deg} \cdot \mathrm{s}^{-2}\right)$} & Int 1: 1212.93 (100.70) & 1 vs. 2 & 0.010 & $5.35(4.21 ; 6.49)$ \\
\hline & Int 2: 868.30 (63.12) & 1 vs. 3 & 0.001 & $6.23(4.94 ; 7.51)$ \\
\hline & Int 3: 735.58 (72.98) & 2 vs. 3 & 0.198 & $1.02(0.45 ; 1.58)$ \\
\hline \multirow{3}{*}{$\begin{array}{c}\text { A MEAN }_{\left(\mathrm{deg} \cdot \mathrm{s}^{-2}\right)}\end{array}$} & Int 1: 701.06 (52.82) & 1 vs. 2 & 0.001 & $6.26(4.96 ; 7.55)$ \\
\hline & Int 2: $488.40(36.71)$ & 1 vs. 3 & $<0.001$ & $7.14(5.69 ; 8.58)$ \\
\hline & Int 3: 368.00 (52.12) & 2 vs. 3 & 0.115 & $0.89(0.33 ; 1.45)$ \\
\hline
\end{tabular}

$\mathrm{A}_{\mathrm{MAX}}$, maximum acceleration in deg. $\mathrm{s}^{-2} ; \mathrm{A}_{\mathrm{MEAN}}$, mean acceleration in deg. $\mathrm{s}^{-2} ; \mathrm{V}_{\mathrm{MAX}}$, maximum velocity in $\mathrm{deg} \cdot \mathrm{s}^{-1} ; \mathrm{V}_{\mathrm{MEAN}}$, mean velocity in deg. $\mathrm{s}^{-1}$. Significant differences for $p$-value $\leq 0.05$ are highlighted in bold.

Table 6. Comparison between load intensity intervals for kinematic parameters in the right shoulder during phase 3 of bench press.

\begin{tabular}{|c|c|c|c|c|}
\hline Parameter & Interval & Comparative & $p$ & $\begin{array}{c}\text { Hedges' } \\
g(95 \% \text { CI })\end{array}$ \\
\hline \multirow{3}{*}{$\mathbf{V}_{\text {MAX }}\left(\operatorname{deg} \cdot \mathrm{s}^{-1}\right)$} & Int 1: 230.37 (11.25) & 1 vs. 2 & $<0.001$ & $5.20(4.08 ; 6.32)$ \\
\hline & Int 2: 172.53 (10.66) & 1 vs. 3 & $<0.001$ & $7.79(6.23 ; 9.35)$ \\
\hline & Int 3: 139.56 (11.72) & 2 vs. 3 & 0.031 & $2.90(2.14 ; 3.66)$ \\
\hline \multirow{3}{*}{$\begin{array}{l}\text { V MEAN }_{\text {MEA }} \\
\left(\text { deg } \cdot \mathrm{s}^{-1}\right)\end{array}$} & Int 1: 139.53 (6.57) & 1 vs. 2 & 0.029 & $4.07(3.13 ; 5.00)$ \\
\hline & Int 2: $106.75(9.11)$ & 1 vs. 3 & $<0.001$ & $7.20(5.74 ; 8.66)$ \\
\hline & Int 3: 88.45 (7.39) & 2 vs. 3 & 1.000 & $2.17(1.50 ; 2.85)$ \\
\hline \multirow{3}{*}{$\mathbf{A}_{\mathbf{M A X}}\left(\mathrm{deg} \cdot \mathrm{s}^{-2}\right)$} & Int 1: 1146.49 (2.51) & 1 vs. 2 & $<0.001$ & $7.11(6.57 ; 8.55)$ \\
\hline & Int 2: 799.73 (67.92) & 1 vs. 3 & $<0.001$ & $9.44(7.58 ; 11.30)$ \\
\hline & Int 3: 660.96 (71.63) & 2 vs. 3 & 0.130 & $1.96(1.31 ; 2.61)$ \\
\hline \multirow{3}{*}{$\begin{array}{c}\text { AMEAN }_{\text {MEA }} \\
\left(\mathrm{deg} \cdot \mathrm{s}^{-2}\right)\end{array}$} & Int 1: 641.74 (51.71) & 1 vs. 2 & $<0.001$ & $3.46(2.62 ; 4.31)$ \\
\hline & Int 2: $436.71(49.54)$ & 1 vs. 3 & $<0.001$ & $6.09(4.82 ; 7.35)$ \\
\hline & Int 3: 320.64 (55.26) & 2 vs. 3 & 0.087 & $2.77(2.02 ; 3.52)$ \\
\hline
\end{tabular}

$\mathrm{A}_{\mathrm{MAX}}$, maximum acceleration in deg $\cdot \mathrm{s}^{-2} ; \mathrm{A}_{\mathrm{MEAN}}$, mean acceleration in $\mathrm{deg} \cdot \mathrm{s}^{-2} ; \mathrm{V}_{\mathrm{MAX}}$, maximum velocity in $\mathrm{deg} \cdot \mathrm{s}^{-1} ; \mathrm{V}_{\text {MEAN }}$, mean velocity in deg. $\mathrm{s}^{-1}$. Significant differences for $p$-value $\leq 0.05$ are highlighted in bold.

\section{Discussion}

This study aimed to analyze BP exercise (BP) right-left shoulder symmetry over different load-intensities in terms of full range of movement, mean and maximum velocities, and accelerations during the two BP's intermediate phases (viz., 2 and 3), in addition to comparing previous kinematics variables between sides. Contrary to the first initial hypothesis, it was found that ROM was different (i.e., higher in left shoulder) between sides over the span of all intensities. All considered velocities and accelerations resulted differently between shoulders but only at highest intensities and during phase 3 . At highest intensities but during phase 2, only velocities were different between sides. Furthermore, at intermediate intensities and during phase 3 only, $V_{\text {MEAN }}$ resulted differently between shoulders. In partial agreement with the second initial hypothesis, both sides' velocities and accelerations decreased with increasing intensity but (i.e., no decrease) from intermediate to highest intensities during both phases 2 and 3. At intermediate intensities, only $\mathrm{V}_{\text {MAX }}$ was different between shoulders and only during phase 3 . As well, in agreement with the second initial hypothesis, ROM — albeit different between sides—did not change across intensities. 
Here, detected velocity decrease over increasing load confirmed at shoulder level what was already shown at the elbow [22]. Regarding different-shoulder acceleration-as a proxy for force-asymmetry, the present study's results only partially confirmed already known electromyography data [24]. Similarly, our outcomes agreed with the findings reported by Pang et al., 2021 [30] who observed no significant differences in shoulder ROM between three different load intervals (40-60-80\% 1RM). Additionally, we found significant differences in ROM between both shoulders at each intensity interval; however, they [30] did not analyze it. It has been very difficult to make further comparisons with other research due to the scarce studies focused on the analysis of ROM between both shoulders in BP exercise.

Our study is the first to use the Xsens system to measure kinematic variables, so it has been impossible to compare our findings with other research using the same measurement technology. During phase 2, it should be noted that the acceleration was positive while the velocity was negative (the subject accelerates the bar to slow it down). Pang et al. [30] found significant differences in angular velocity when comparing $40 \%$ vs. $80 \% 1 \mathrm{RM}$ during shoulder extension $(p<0.05)$, coinciding with our results when comparing intervals 1,2 , and 3 during phase 2 . To the best of our knowledge, no further research exists that has compared accelerations and angular velocities between shoulders during BP. The analysis of joint kinematics may be useful to prevent injuries and improve athletes' performance [7]; moreover, weightlifters commonly suffer shoulder injuries [8] since this joint is exposed to repetitive stresses and high weights during BP, as well as connective tissue changes and joint instability [9]. Despite this, the knowledge of shoulder kinematics might provide key results to direct injury prevention strategies. Thus, our study supposes a starting point for the analysis of shoulder joint kinematics during BP. Nevertheless, future research should be conducted to confirm or discuss our results, allowing a wider and richer approach in this matter.

Nevertheless, some limitations should be considered in the present study. The sample size was rather small, which increases the difficulty of generalizing of results. Thus, it would be interesting to carry out a similar study with a larger sample to confirm the obtained outcomes. Furthermore, the distribution of load intensity intervals was done a posteriori and was too wide. Therefore, although participants were instructed to push the barbell as fast as possible during the concentric phase of every trial, future investigations should consider establishing the intensity intervals a priori and selecting smaller ranges of load intensity. Moreover, the sticking region was not considered in our analysis. It could be interesting if future studies consider this point due to its importance for performance, especially with heavy loads.

In summary, this study fills a knowledge gap regarding simple kinematics description of BP. Here, the shown data can provide reference data to strength training professionals to better read test results and better design training programs.

\section{Conclusions}

Based on ROM and velocity outcomes, we conclude that there exists a kinematics asymmetry between both shoulders during phases 2 and 3 of bench press, although the acceleration was similar during both phases at all load intensities. Similarly, kinematic parameters differ between loads of $55-75 \%$ RM compared to $75-100 \%$ RM loads.

This study provides information about the need to unilaterally assess upper limbs during overload bilateral exercises to detect possible disbalances or asymmetries. It may be especially relevant in sports disciplines where the bench press exercise determines success during competition, such as Powerlifting or Paralympics Weightlifting.

Author Contributions: Conceptualization, M.R. and J.P.-G.; methodology, M.R. and J.M.F.-G.; software, R.G.-H.; validation, K.G., J.M.F.-G. and M.R.; formal analysis, J.M.F.-G. and J.C.-V.; investigation, J.M.F.-G.; resources, K.G.; data curation, R.G.-H.; writing-original draft preparation, J.M.F.-G., J.C.V., H.N. and L.P.A.; writing-review and editing, J.M.F.-G., J.C.-V. and L.P.A.; visualization, J.C.-V., 
H.N. and J.P.-G.; supervision, K.G., J.P.-G. and L.P.A.; project administration, K.G. and J.P.-G. All authors have read and agreed to the published version of the manuscript.

Funding: This research received no external funding.

Institutional Review Board Statement: The study was conducted according to the guidelines of the Declaration of Helsinki, and approved by the Bioethics and Biosafety Committee of the University of Extremadura (protocol code: 126/2018).

Informed Consent Statement: Informed consent was obtained from all subjects involved in the study.

Data Availability Statement: The datasets used during the current study are available from the corresponding author on reasonable request.

Conflicts of Interest: The authors declare no conflict of interest.

\section{References}

1. Michal, W.; Michal, K.; Marcin, B. The Influence of Compressive Gear on Maximal Load Lifted in Competitive Powerlifting. Biol. Sport 2020, 37, 437-441. [CrossRef] [PubMed]

2. Schoenfeld, B.J.; Contreras, B.; Vigotsky, A.D.; Ogborn, D.; Fontana, F.; Tiryaki-Sonmez, G. Upper Body Muscle Activation during Low-versus High-Load Resistance Exercise in the Bench Press. Isokinet. Exerc. Sci. 2016, 24, 217-224. [CrossRef]

3. Speranza, M.J.A.; Gabbett, T.J.; Johnston, R.D.; Sheppard, J.M. Effect of Strength and Power Training on Tackling Ability in Semiprofessional Rugby League Players. J. Strength Cond. Res. 2016, 30, 336-343. [CrossRef]

4. Gregory, H.G.; Travis, T.N. Essentials of Strength Training and Conditioning, 4th ed.; Human Kinetics: Champaign, IL, USA, 2015, ISBN 978-1-4925-0162-6.

5. Krzysztofik, M.; Golas, A.; Wilk, M.; Stastny, P.; Lockie, R.G.; Zajac, A. A Comparison of Muscle Activity Between the Cambered and Standard Bar During the Bench Press Exercise. Front. Physiol. 2020, 11, 875. [CrossRef]

6. Kolber, M.J.; Beekhuizen, K.S.; Cheng, M.-S.S.; Hellman, M.A. Shoulder Joint and Muscle Characteristics in the Recreational Weight Training Population. J. Strength Cond. Res. 2009, 23, 148-157. [CrossRef]

7. Huang, Y.-P.; Chou, Y.-L.; Chen, F.-C.; Wang, R.-T.; Huang, M.-J.; Chou, P.P.-H. Elbow Joint Fatigue and Bench-Press Training. J. Athl. Train. 2014, 49, 317-321. [CrossRef]

8. Aasa, U.; Svartholm, I.; Andersson, F.; Berglund, L. Injuries among Weightlifters and Powerlifters: A Systematic Review. Br. J. Sports Med. 2017, 51, 211-219. [CrossRef] [PubMed]

9. Kolber, M.J.; Corrao, M.; Hanney, W.J. Characteristics of Anterior Shoulder Instability and Hyperlaxity in the Weight-Training Population. J. Strength Cond. Res. 2013, 27, 1333-1339. [CrossRef] [PubMed]

10. García-Ramos, A.; Pestaña-Melero, F.L.; Pérez-Castilla, A.; Rojas, F.J.; Gregory Haff, G. Mean Velocity vs. Mean Propulsive Velocity vs. Peak Velocity: Which Variable Determines Bench Press Relative Load With Higher Reliability? J. Strength Cond. Res. 2018, 32, 1273-1279. [CrossRef]

11. Carzoli, J.P.; Sousa, C.A.; Belcher, D.J.; Helms, E.R.; Khamoui, A.V.; Whitehurst, M.; Zourdos, M.C. The Effects of Eccentric Phase Duration on Concentric Outcomes in the Back Squat and Bench Press in Well-Trained Males. J. Sports Sci. 2019, 37, 2676-2684. [CrossRef]

12. Cronin, J.B.; McNair, P.J.; Marshall, R.N. Force-Velocity Analysis of Strength-Training Techniques and Load: Implications for Training Strategy and Research. J. Strength Cond. Res. 2003, 17, 148-155. [CrossRef]

13. Dugdale, J.H.; Hunter, A.M.; Di Virgilio, T.G.; Macgregor, L.J.; Hamilton, D.L. Influence of the "Slingshot" Bench Press Training Aid on Bench Press Kinematics and Neuromuscular Activity in Competitive Powerlifters. J. Strength Cond. Res. 2019, 33, 327-336. [CrossRef]

14. Elliott, B.; Wilson, G.; Kerr, G. A Biomechanical Analysis of the Sticking Region in the Bench Press. Med. Sci. Sports Exerc. 1989, 21, 450-462. [CrossRef] [PubMed]

15. Rahmani, A.; Rambaud, O.; Bourdin, M.; Mariot, J.-P. A Virtual Model of the Bench Press Exercise. J. Biomech. 2009, 42, 1610-1615. [CrossRef] [PubMed]

16. Sakamoto, A.; Kuroda, A.; Sinclair, P.J.; Naito, H.; Sakuma, K. The Effectiveness of Bench Press Training with or without Throws on Strength and Shot Put Distance of Competitive University Athletes. Eur. J. Appl. Physiol. 2018, 118, 1821-1830. [CrossRef]

17. Bartolomei, S.; Nigro, F.; Ruggeri, S.; Malagoli Lanzoni, I.; Ciacci, S.; Merni, F.; Sadres, E.; Hoffman, J.R.; Semprini, G. Comparison Between Bench Press Throw and Ballistic Push-up Tests to Assess Upper-Body Power in Trained Individuals. J. Strength Cond. Res. 2018, 32, 1503-1510. [CrossRef] [PubMed]

18. Tillaar, R. van den Comparison of Kinematics and Muscle Activation between Push-up and Bench Press. Sports Med. Int. Open 2019, 3, E74-E81. [CrossRef]

19. Castro, A.H.; Zangakis, D.; Moir, G.L. The Effects of Accentuated Eccentric Loading on Mechanical Variables and Agonist Electromyography during the Bench Press. Sports 2020, 8, 79. [CrossRef] [PubMed]

20. Król, H.; Gołaś, A. Effect of Barbell Weight on the Structure of the Flat Bench Press. J. Strength Cond. Res. 2017, 31, 1321-1337. [CrossRef] 
21. Mausehund, L.; Werkhausen, A.; Bartsch, J.; Krosshaug, T. Understanding Bench Press Biomechanics-The Necessity of Measuring Lateral Barbell Forces. J. Strength Cond. Res. 2021, in press. [CrossRef]

22. Ojasto, T.; Häkkinen, K. Effects of Different Accentuated Eccentric Loads on Acute Neuromuscular, Growth Hormone, and Blood Lactate Responses during a Hypertrophic Protocol. J. Strength Cond. Res. 2009, 23, 946-953. [CrossRef]

23. Larsen, S.; Gomo, O.; van den Tillaar, R. A Biomechanical Analysis of Wide, Medium, and Narrow Grip Width Effects on Kinematics, Horizontal Kinetics, and Muscle Activity on the Sticking Region in Recreationally Trained Males During 1-RM Bench Pressing. Front. Sports Act. Living 2021, 2, 229. [CrossRef] [PubMed]

24. Krzysztofik, M.; Jarosz, J.; Matykiewicz, P.; Wilk, M.; Bialas, M.; Zajac, A.; Golas, A. A Comparison of Muscle Activity of the Dominant and Non-Dominant Side of the Body during Low versus High Loaded Bench Press Exercise Performed to Muscular Failure. J. Electromyogr. Kinesiol. 2021, 56, 102513. [CrossRef] [PubMed]

25. Saeterbakken, A.H.; Solstad, T.E.J.; Behm, D.G.; Stien, N.; Shaw, M.P.; Pedersen, H.; Andersen, V. Muscle Activity in Asymmetric Bench Press among Resistance-Trained Individuals. Eur. J. Appl. Physiol. 2020, 120, 2517-2524. [CrossRef]

26. Roetenberg, D.; Luinge, H.; Slycke, P. Xsens MVN: Full 6DOF Human Motion Tracking Using Miniature Inertial Sensors; Xsens Motion Technologies BV: Enschede, The Netherlands, 2009; Volume 3.

27. Woltring, H.J. A Fortran Package for Generalized, Cross-Validatory Spline Smoothing and Differentiation. Adv. Eng. Softw. (1978) 1986, 8, 104-113. [CrossRef]

28. Poitras, I.; Dupuis, F.; Bielmann, M.; Campeau-Lecours, A.; Mercier, C.; Bouyer, L.J.; Roy, J.-S. Validity and Reliability of Wearable Sensors for Joint Angle Estimation: A Systematic Review. Sensors 2019, 19, 1555. [CrossRef]

29. Hopkins, W.G.; Marshall, S.W.; Batterham, A.M.; Hanin, J. Progressive Statistics for Studies in Sports Medicine and Exercise Science. Med. Sci. Sports Exerc. 2009, 41, 3-12. [CrossRef] [PubMed]

30. Pang, B.; Ji, Z.; Zhang, Z.; Sun, Y.; Ma, C.; He, Z.; Hu, X.; Jiang, G. Strength Training Characteristics of Different Loads Based on Acceleration Sensor and Finite Element Simulation. Sensors 2021, 21, 647. [CrossRef] [PubMed] 